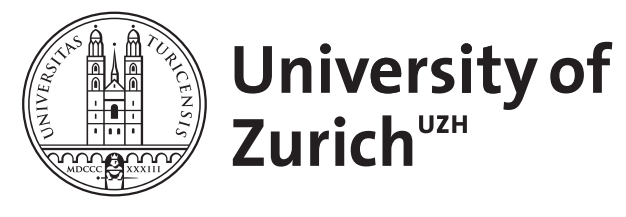

\title{
Sprayer for quantitative application of odor stimuli
}

El-Sayed, Ashraf ; Gódde, Josef ; Arn, Heinrich

\begin{abstract}
A novel device is described for the quantitative application of chemical stimuli. The device uses ultrasound to disperse a solution of volatile chemicals as an aerosol. A motor-driven syringe controls the rate at which the solution is released from a glass capillary. Vibration of the capillary disperses the released solution into microdroplets that evaporate completely within a few centimeters of the tip. The ratio of chemical stimulus to solvent is maintained until the liquid is dispersed and therefore the release rate of the chemical stimulus can be set and calculated straightforwardly from the dilution factor and the dynamically controllable speed of the syringe plunger. The sprayer permits the delivery of chemical stimulus independent of relative vapor pressures of the components and of environmental factors such as temperature. The sprayer is easy to operate, can be constructed from inexpensive materials, and can be used to emit odor stimuli in the wind tunnel or any other bioassay for pheromones and plant volatiles
\end{abstract}

DOI: https://doi.org/10.1093/ee/28.6.947

Posted at the Zurich Open Repository and Archive, University of Zurich

ZORA URL: https://doi.org/10.5167/uzh-153847

Journal Article

Published Version

Originally published at:

El-Sayed, Ashraf; Gódde, Josef; Arn, Heinrich (1999). Sprayer for quantitative application of odor stimuli. Environmental Entomology, 28(6):947-953.

DOI: https://doi.org/10.1093/ee/28.6.947 


\title{
Sprayer for Quantitative Application of Odor Stimuli
}

\author{
ASHRAF EL-SAYED, ${ }^{1,2}$ JOSEF GÖDDE, ${ }^{3}$ AND HEINRICH ARN ${ }^{2}$
}

\begin{abstract}
Environ. Entomol. 28 (6): 947-953 (1999)
ABSTRACT A novel device is described for the quantitative application of chemical stimuli. The device uses ultrasound to disperse a solution of volatile chemicals as an aerosol. A motor-driven syringe controls the rate at which the solution is released from a glass capillary. Vibration of the capillary disperses the released solution into microdroplets that evaporate completely within a few centimeters of the tip. The ratio of chemical stimulus to solvent is maintained until the liquid is dispersed and therefore the release rate of the chemical stimulus can be set and calculated straightforwardly from the dilution factor and the dynamically controllable speed of the syringe plunger. The sprayer permits the delivery of chemical stimulus independent of relative vapor pressures of the components and of environmental factors such as temperature. The sprayer is easy to operate, can be constructed from inexpensive materials, and can be used to emit odor stimuli in the wind tunnel or any other bioassay for pheromones and plant volatiles.
\end{abstract}

KEY WORDS pheromones, plant volatiles, odor quantification, ultrasound, release rate, olfactory stimulation

IN STUDIES OF olfaction, the quantitative application of volatile stimuli has been a long-standing problem. Quantitative odor stimulation requires that a gas, mostly air, contains a known concentration of a chemical stimulus. The high sensitivity of many olfactory organs necessitates the use of very low concentrations of the stimulus. In studies with insects, the stimulus is often administered using a substrate such as a filter paper, a rubber stopper, or polyethylene caps. The amount of test substance released per unit of time and carried with an air stream to the test animal depends on parameters such as vapor pressure, the affinity of test chemicals for substrate, and temperature. Depending on the substrate used in the behavior bioassay, dose-response curves for the same pheromone blend can differ by $>2$ log units (Linn et al. 1984, Sanders 1990). The sensitivity of insects to stimuli using these techniques can at the best be expressed on relative scales, unless the stimuli are quantified using techniques such as radioactive labeling (Kaissling 1985) or they are collected on adsorbents followed by analysis (Cross 1980). Better control over the stimulus concentration is obtained with another approach, in which the test substance is allowed to equilibrate between a reservoir of a solvent-odorant mixture and a closed gas space (Färbert et al. 1997). Using this method, gas concentrations are still dependent on factors such as temperature and the diluting solvent,

\footnotetext{
${ }^{1}$ Department of Zoology, University of Zürich CH-8057 Zürich, Switzerland. Current address: Southern Crop Protection \& Food Research Centre Agriculture \& Agri-Food Canada, Vineland Station, ON, Canada L0R 2E0.

${ }^{2}$ To whom all correspondence should be addressed: Swiss Federal Research Station (FAW), CH-8820 Wädenswil, Switzerland.

${ }^{3}$ Steinklepper Weg 1, D-35753 Greifenstein, Germany.
}

which are difficult to control for components of different vapor pressures or polarities.

Recently, pressurized canisters were used for dispersing pheromone in the field as aerosols for disruption of sex pheromone communication. The machines contain timer-activated mechanisms for releasing puffs of pheromone components. This allows the release pheromones only during the flight period and avoids unnecessary emission during the day where the insects are inactive. The pheromone chemicals in these canisters are protected from oxidative degradation until after it is released into the air (Shorey and Gerber 1996, Shorey et al. 1996).

In this article we describe a device capable of releasing predetermined amounts of semiochemicals in constant proportions, chemical and isomeric purity. The sprayer makes it possible to emit pheromone blends at precise and biologically meaningful rates (picograms or femtograms per minute). The sprayer was developed to characterize the pheromone blend of the grapevine moth, Lobesia botrana Denis \& Schiffermüller, (El-Sayed 1996, 1998a, 1999a) and it has been successfully used with other species, e.g., codling moth, Cydia pomonella L. (El-Sayed et al. 1998b, $1999 \mathrm{~b})$, the pea midge, Contarinia pisi Winnertz (Y. Hillbur and E.-S., unpublished data) and the North American grape berry moth, Endopiza viteana Clemens, (R. M. Trimble, personal communication). In addition to pheromone studies, the sprayer can also be used in studies of plant volatiles. We review the construction, accuracy of chemical stimuli emitted from the sprayer, and bioassays to investigate the effect of ultrasound and solvent emitted during evaporation on male behavior. 
Precise syringe pump

(known flow rate, e. g. $10 \mathrm{ul} / \mathrm{min}$ )

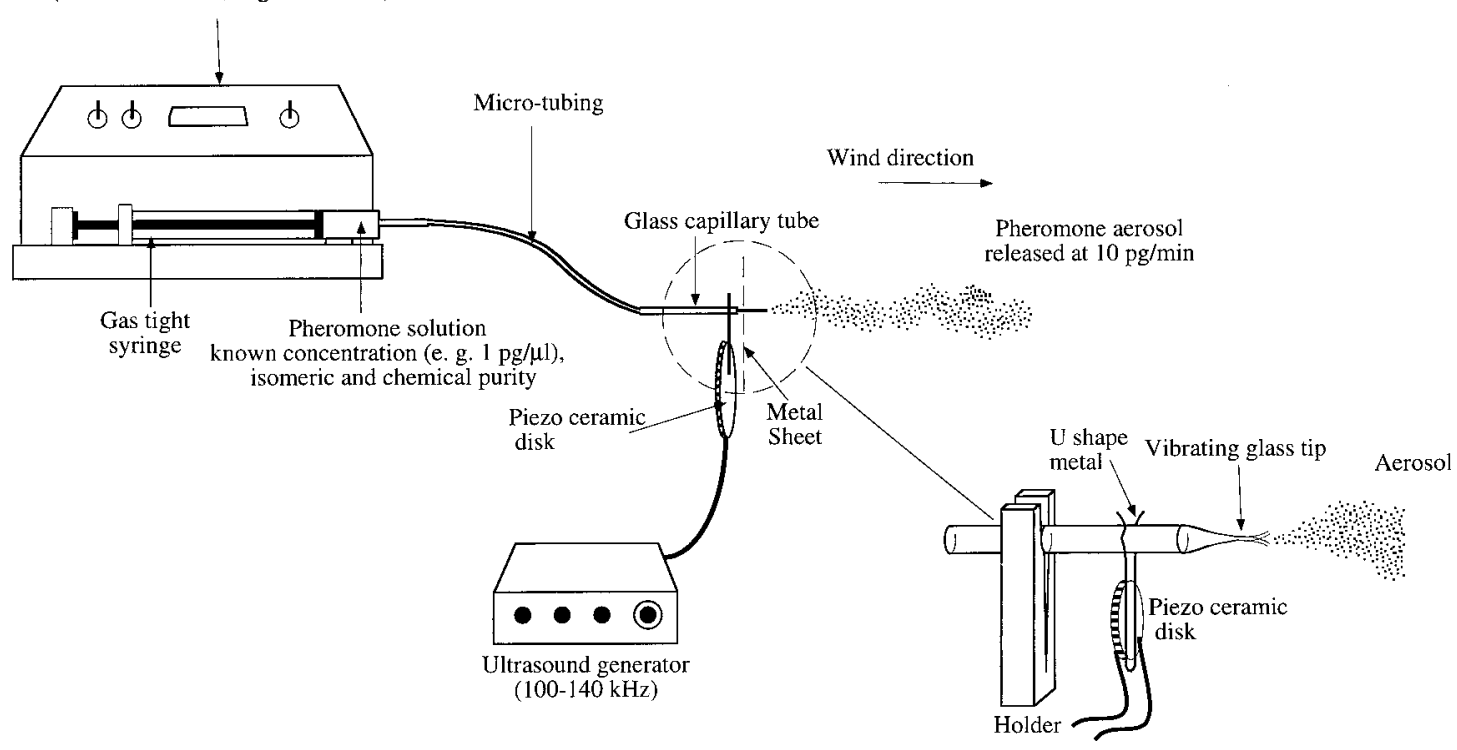

Fig. 1. Sprayer set-up used in the wind tunnel experiments.

\section{Materials and Methods}

Operating Principles. The sprayer relies on 2 basic ideas: first, the odorant is diluted in a solvent that has been shown to have no behavioral effect on the species of interest. Second, this odorant solution is released into the air at a known rate. The odorant solution is released using a motor-driven syringe that pushes the odorant solution to the tip of a glass capillary with an opening of $10-50 \mu \mathrm{m}$ i.d. at a rate of $\approx 10$ $\mu \mathrm{l} / \mathrm{min}$ (Fig. 1). The capillary tip is excited to oscillate at $\approx 120 \mathrm{kHz}$, or a harmonic of this frequency. At $\approx 25$ $\mu \mathrm{m}$ amplitude peak-to-peak, an oscillation of $120 \mathrm{kHz}$ creates a peak tip velocity of $9.42 \mathrm{~ms}^{-1}$, and a peak tip acceleration of $7.1 \times 10^{6} \mathrm{~ms}^{-2}$. This releases 1 or 2 microdroplets of odorant solution at each oscillation and results in an aerosol of the solution that disperses and evaporates completely within $<5 \mathrm{~cm}$ from the capillary tip when placed in a wind tunnel under the bioassay conditions (Fig. 2). The release rate of the test chemicals can be controlled by 2 independent parameters: (1) the concentration of the odorant solution, and (2) the flow rate generated by the syringe pump. Both of these parameters can be quantitatively adjusted and therefore the amount of odorant released per unit of time can be precisely calculated.

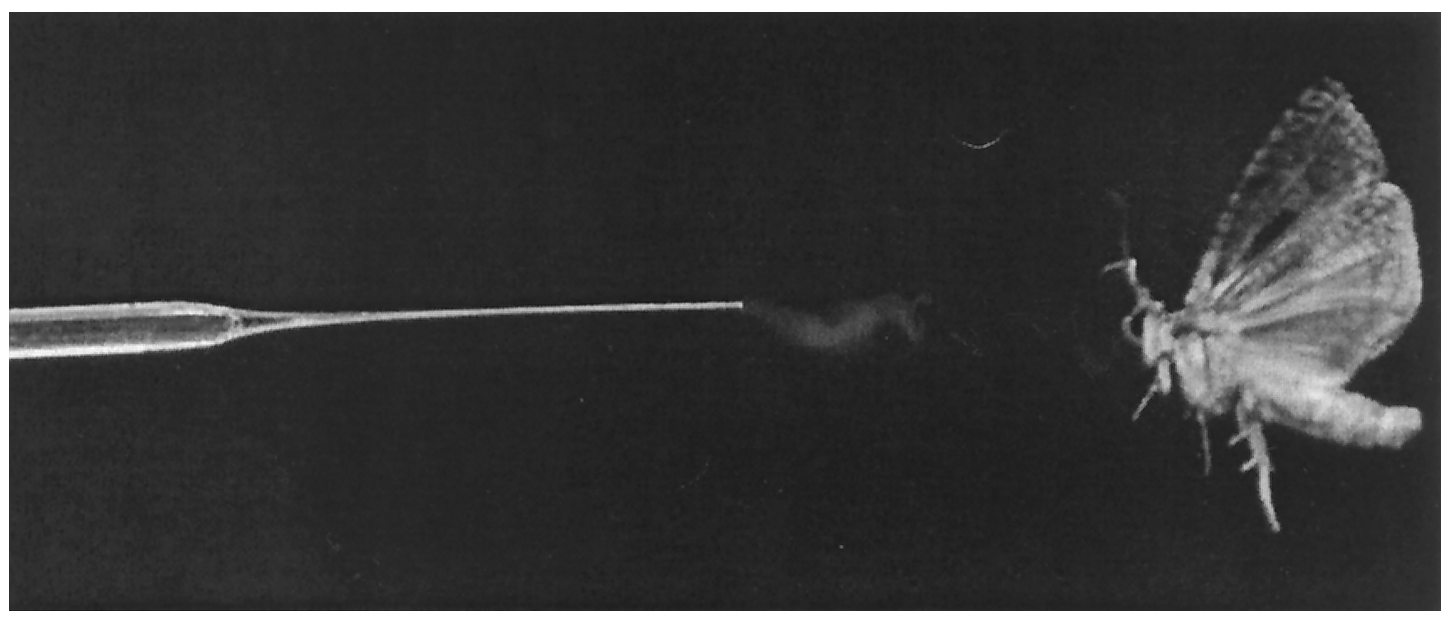

Fig. 2. Photograph of the active sprayer in the wind tunnel and a male codling moth, C. pomonella released from $\approx 2 \mathrm{~m}$ approaching the tip of the capillary tube. 
Construction. The method of exciting the tip of a glass capillary tip to vibrate at ultrasonic frequencies was developed by Gödde (1986). A piezo disk of 10-25 mm o.d. and $\approx 1-2 \mathrm{~mm}$ thickness with solderable silver coatings on both faces (Cat. Nr. 4322 020, Valvo, Hamburg, Germany) is driven at its bending mode resonance by a sine or square wave of $\approx 20 \mathrm{~V}$ peak to peak. The driving signals were taken from standard function generators (FG-8002 GoldStar Precision, Seoul, Korea). A U-shaped wire $1 \mathrm{~mm}$ diameter is soldered to one face of the piezo disk so that the open stems reach $\approx 10 \mathrm{~mm}$ over the rim of the disk (Fig. 1, inset). This wire serves 2 purposes: it holds the piezo disk in place by spring force and transfers the oscillations to the glass capillary tube. The tip of the capillary is an acoustical transformer, and therefore the amplitude of oscillation increases toward the end, where it may reach $100 \mu \mathrm{m}$. Glass capillaries of $1 \mathrm{~mm}$ o.d. and $0.4 \mathrm{~mm}$ i.d. (Narishige, London) were drawn out using an electrode puller (Narishige) and broken to tip diameters of $\approx 55 \mu \mathrm{m}$ o.d. $/ 40 \mu \mathrm{m}$ i.d. (determined by scanning electron microscopy). The geometry of the capillary tip may vary considerably between individual capillaries without impairing their function. If oscillation frequencies at higher harmonics of the fundamental bending resonance frequency (usually in the range of $120 \mathrm{kHz}$ ) are intended (see Results and Discussion), the capillaries should have long, slender tips with $>4 \mathrm{~mm}$ tip range of $<100 \mu \mathrm{m}$ i.d. Using such capillaries, vaporization was achieved at frequencies of up to $1 \mathrm{MHz}$. The frequency being adjusted visually to the resonance of the individual capillary. A microinjection pump (CMA/100, Carnegie Medicine AB, Stockholm, Sweden) was used to deliver the odorant solution via micro tubing, $1 \mathrm{~m}$ long and $0.12 \mathrm{~mm}$ i.d. (CMA/Microdialysis, Carnegie Medicine AB, Stockholm, Sweden) to the capillary tube (Fig. 1). A tubing adapter (CMA/Microdialysis, Carnegie Medicine) was used to connect the micro tubing to the syringe outlet and to the capillary tube inlet.

The flow rate $(d V / d t)$ of the odorant solution is determined by the speed of the syringe piston. According to the law of Poiseuille (Adam et al. 1977), a given flow rate of odorant solution builds up an odorant solution-head pressure $(\Delta P)$, which is proportional to the odorant solution flow rate: $d V / d t=\Delta P \times$ $C$. For cylindrical tubes of luminal radius $R$ and length $L, \Delta P$ depends on the viscosity $\eta$ of the fluid according to $C=\sim R^{4} /(8 \eta L)$. The narrowest part in the tubing determines $C$. This is in most cases the capillary tip. Hence, $\Delta P$ depends on the viscosity of the odorant solution and even more so on the geometry of the capillary tip. The viscosity of the odorant solution is almost identical with the viscosity of the solvent if the odorant is highly diluted.

Wind Tunnel Experiments. The 1st test of the sprayer for releasing pheromones was carried out in the wind tunnel of Witzgall and Arn (1991). The capillary tube and piezo ceramic disk were installed at the upwind end of the tunnel behind a perforated metal sheet with $3 \mathrm{~mm}$ holes. The tip was oriented at the center aperture of the metal sheet and projected $\approx 1.5 \mathrm{~cm}$ into the wind tunnel (Fig. 1). It was protected from approaching males using a glass cylinder ( 10 by $10 \mathrm{~cm}$ ) with mesh at it is distal end. The pump and function generator were kept outside the tunnel. The light intensity was 30 lux at the center of the tunnel, wind speed was $30 \mathrm{~cm} / \mathrm{s}$ and air temperature was $22 \pm$ $2^{\circ} \mathrm{C}$. Twenty-five 2 - to 3 -d-old $L$. botrana males were immobilized at $4^{\circ} \mathrm{C}$ and transferred individually to glass release tubes ( 15 by $2.5 \mathrm{~cm}$ ). Bioassays commenced at the beginning of the scotophase and continued for $4 \mathrm{~h}$. Males were released individually in the wind tunnel and the following behavioral responses were recorded: activation = wing fanning, random walking in the release tube; upwind flight $=$ beginning of upwind flight for at least 10-15 $\mathrm{cm}$; landing = touchdown at the source. Differences in the number of males completing successive stages in their advance toward the source were compared using chi-square analysis $(2 \times 2)$ with Yates correction (GraphPad 1993).

Recording Flight Behavior. The flight behavior of male $L$. botrana to females without and with different ultrasound frequencies was recorded using 2 couple charge device (CCD) cameras (aqua tv HR 480, Henz Memo AG, Suhr, Switzerland) (El-Sayed et al. 1999c). The 2 cameras were placed on the side of the wind tunnel, $\approx 120 \mathrm{~cm}$ from the tunnel and $105 \mathrm{~cm}$ from the floor. Their axis formed an angle of $\approx 90^{\circ}$. The sectors covered by the 2 cameras overlapped over a space that had a maximum length of $50 \mathrm{~cm}$ along the tunnel axis. The lateral side of the wind tunnel opposite to the 2 cameras was illuminated using a light box. Analysis of flight tracks was done off-line. A Macintosh program (TrackEvaluat8) was used to view and analyze the following flight parameters: straightness $=$ length of the net path traveled/the total distance traveled; linear velocity $=$ total distance traveled on longitudinal axis/elapse time; track angle $=$ the deviation of the observed flight path from the wind line. Grand mean was calculated for each of the 3 parameters to each of the pheromone source tested. The means were compared using one-way analysis of variance (ANOVA) (Abacus Concepts 1995).

Chemicals. The synthetic pheromones tested were obtained from the laboratory of H.A. The chemical purity of the compounds was $93-99.9 \%$. The odorant solutions were made by mixing the appropriate quantities of synthetic compounds in high-performance liquid chromatography (HPLC) grade ethanol. A given odorant solution was typically used for $\approx 100$ min and expelled at $10 \mu \mathrm{l} / \mathrm{min}$.

Effect of Ultrasound and Solvent. The possible effects of ultrasound emission and of the solvent ethanol on the response of male L. botrana were tested. The response of males to calling females was compared with and without the presence of ultrasound and solvent. The sprayer and the female cage were separated by $2 \mathrm{~cm}$. The sprayer was operated at 3 frequencies without solvent; ethanol was evaporated at $100 \mathrm{kHz}$ and a delivery rate of $10 \mathrm{pg} / \mathrm{min}$. Calling females in the absence of ultrasound served as control. 
Table 1. Response of $L$. botrana males to calling females without and with ultrasound at 3 frequencies and ultrasound plus solvent

\begin{tabular}{lccccrr}
\hline \multicolumn{1}{c}{ Source $^{a}$} & $\begin{array}{c}\text { Frequency, } \\
\mathrm{kHz}\end{array}$ & $\begin{array}{c}\text { Landing, } \\
\%\end{array}$ & No. tracks & $\begin{array}{c}\text { Straightness, } \\
\text { rel. unit }{ }^{b}\end{array}$ & $\begin{array}{c}\text { Linear velocity, } \\
\mathrm{cm}^{\prime} \mathrm{s}^{c}\end{array}$ & $\begin{array}{c}\text { Track angle, } \\
\left({ }^{\circ}\right)^{d}\end{array}$ \\
\hline Females & 0 & 98 & 45 & $0.62 \pm 0.16$ & $22.2 \pm 0.83$ & $49.5 \pm 11.0$ \\
Females & 10 & 95 & 48 & $0.60 \pm 0.21$ & $24.7 \pm 09.2$ & $51.1 \pm 13.8$ \\
Females & 100 & 97 & 39 & $0.60 \pm 0.20$ & $22.8 \pm 09.2$ & $50.3 \pm 14.2$ \\
Females & 1,000 & 96 & 45 & $0.57 \pm 0.21$ & $22.6 \pm 08.3$ & $53.1 \pm 10.2$ \\
Females + Ethanol & 100 & 98 & 45 & $0.60 \pm 0.20$ & $22.8 \pm 08.3$ & $51.5 \pm 12.5$ \\
\hline
\end{tabular}

Flight parameters are means $\pm \mathrm{SD}$, no significant differences were found between values in the same column; $P \leq 0.05$, chi-square test (InStat 1993) for percent landing, and ANOVA for flight parameters (StatView 1995). Fifty males were tested for each treatment.

${ }^{a}$ Two calling females were used.

${ }^{b} F=0.541 ; \mathrm{df}=4,217 ; P=0.7054$

${ }^{c} F=0.603 ; \mathrm{df}=4,217 ; P=0.6609$.

${ }^{d} F=0.317 ; \mathrm{df}=4,217 ; P=0.8711$

Accuracy of Chemical Stimuli Emitted by the Sprayer. A hexane solution containing $100 \mathrm{ng} / \mu \mathrm{l}$ of dodecyl acetate (12Ac) and $100 \mathrm{ng} / \mu \mathrm{l}$ octadecyl acetate (18Ac) in 1:1 ratio was allowed to evaporate from the same capillary tube at a rate of $10 \mu \mathrm{l} / \mathrm{min}$ (i.e., $1 \mu \mathrm{g}$ for each compound per minute). The aerosol that formed at the tip of the capillary tube was trapped for $1 \mathrm{~min}$ on a Pasteur pipette, which was then eluted with $10 \mu \mathrm{l}$ of hexane. This process was continued for 8 successive minutes. The eluted solution was injected immediately into Carlo Erba gas-chromatograph equipped with a 30 by $0.32 \mathrm{~mm}$ i.d. DB- 5 column (J\&W Scientific, Folsom, CA). The temperature was programmed from $40^{\circ} \mathrm{C}$ (1 min hold), at $25^{\circ} \mathrm{C} / \mathrm{min}$ to $150^{\circ} \mathrm{C}, 5^{\circ} \mathrm{C} / \mathrm{min}$ to $210^{\circ} \mathrm{C}$ and $25^{\circ} \mathrm{C} / \mathrm{min}$ to $250^{\circ} \mathrm{C}$. Helium was used as the carrier gas at a linear flow velocity of $35 \mathrm{~cm} / \mathrm{s}$.

Measuring Pheromone Concentration. Using the sprayer, the odorant solution is released from the capillary tip as an aerosol of microdroplets. If parts of these microdroplets do not evaporate while carried with the air stream moving toward the downwind end of the tunnel, the heavier microdroplets will fall down to the floor of the wind tunnel. This would lead to a decline in the pheromone concentration at the downwind end of the tunnel. To measure the pheromone concentration at different distances from the capillary tip, a portable electroantennogram (EAG) recording system (see Sauer et al. 1992; Färbert et al. 1997) with antennae from 2- to 3-d-old male L. botrana was used. The pheromone plume of (E,Z)-7,9-dodecadienyl acetate $(\mathrm{E} 7, \mathrm{Z} 9-12 \mathrm{Ac})$ generated from the sprayer at a release rate of $1000 \mathrm{pg} / \mathrm{min}$ in the wind tunnel was investigated horizontally $(20 \mathrm{~cm})$ at 50 and $166 \mathrm{~cm}$ from the capillary tip. The EAG system was moved horizontally through the pheromone plume using an adjustable mobile stand.

Ability of the Sprayer to Release Different Concentrations. Four-fold dilutions of the 3-component blend (El-Sayed et al. 1999a) in the ratio $10 \mathrm{pg} / \mathrm{min} \mathrm{E} 7, \mathrm{Z} 9-$ 12Ac, $\quad 0.5 \mathrm{pg} / \mathrm{min}$ of $(E, Z)-7,9-$ dodecadien-1-ol $(\mathrm{E} 7, \mathrm{Z} 9-12 \mathrm{OH})$ and $0.1 \mathrm{pg} / \mathrm{min}$ of $(Z)$-9-dodecenyl acetate (Z9-12Ac) were prepared. A 1- $\mu$ l sample from a given dilution contained, $0.01,0.1,1,10$, and $100 \mathrm{pg}$ of E7,Z9-12Ac. Seventy-five males were tested for each release rate.
Ability of the Sprayer to Release Pheromone Blends with Proportions of Components. Males were flown to a 2-component blend containing a constant amount of the main pheromone component, E7,Z9-12Ac (10 pg/ min) plus varying amounts of the minor pheromone component, E7,Z9-12OH (i.e., 0, 0.02, 0.1, 0.5, 2, and $10 \mathrm{pg} / \mathrm{min}$ ). One hundred males were tested for each blend.

\section{Results and Discussion}

Effect of Ultrasound and Solvent. The response of L. botrana males to calling females, to calling females plus 3 frequencies of ultrasound, and to calling females plus ethanol aerosol was similar (Table 1). Not only was the ultrasound without effect on the males, the identical attractiveness of females with and without ultrasound demonstrated that the ultrasound had no effect on the female's ability to attract males. Thus, neither the ultrasound produced nor the solvent ethanol appear to have an effect on male upwind flight or female calling. Although ultrasound did not appear to affect the behavior of male or female L. botrana, this device should be used with caution in other insect species. Insect traps emitting ultrasound are avoided by several species of lepidoptera (Treat 1962). Ultrasound can abruptly arrest pheromone-induced behavior such as wing fanning and upwind flight (Baker and Cardé 1978). A solution to this problem could be to operate the sprayer at the highest harmonic frequency effective for dispersing the odorant solution. A range of $1 \mathrm{MHz}$ would be well above the upper limit of acoustic perception of most animals. In addition to $L$. botrana, the sprayer has been tested with the following species, E. viteana, C. pomonella, the grape moth, Eupoecilia ambiguella Hübner, the pea midge, C. pisi, with no apparent affect of either ultrasound or the solvent on male behavior. We recommend the user carry out a similar experiment before using the sprayer with a new species.

Little is known about the influence of the solvent on insect behavior. With ethanol or hexane at the release rate of $10 \mu \mathrm{l} / \mathrm{min}$, no effect on behavior of males or females was apparent in all tested species so far. But, there are possible indirect effects of the solvents that should be regarded when designing experiments. 


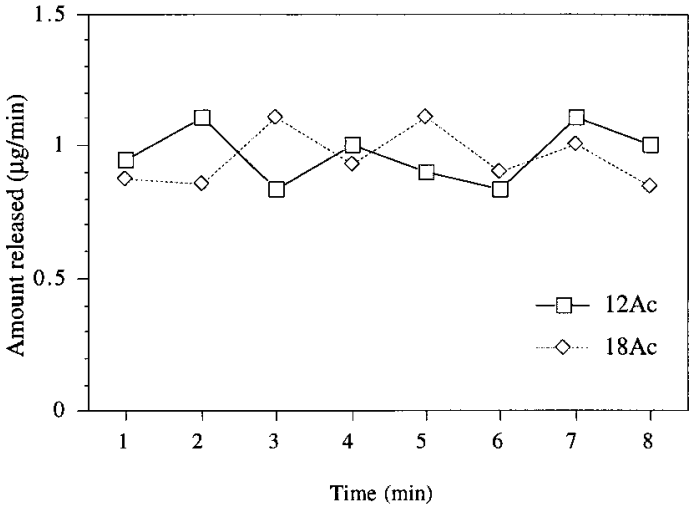

Fig. 3. Amount of $12 \mathrm{Ac}$ and $18 \mathrm{Ac}$ released by the sprayer for 8 successive minutes as determined by aerosol collection and GC analysis.

With highly diluted odorant, the adsorption of the odorant to the interior surfaces of syringe, tubing, and capillary could significantly diminish the original odorant-concentration in the bulk odorant solvent mixture. The ratio of adsorbed odorant versus odorant in the bulk-solution depends on the solvent. Hence a polar odorant that is soluble in a polar solvent such as water or ethanol, as well as in a much less polar solvent such as hexane, may be adsorbed to polar surfaces only when prepared in nonpolar solvent. The resulting deviation of the real concentration of the odorant in the odorant solvent mixture from the calculated concentration depends on the adsorption-capacity of the delivery system.

Accuracy of Chemical Stimuli Emitted by the Sprayer. Gas chromatography analysis of the aerosol collected at the capillary tip indicated that the average amount released per minute was $0.96 \mu \mathrm{g} \pm 0.1$ for $12 \mathrm{Ac}$ and $0.94 \mu \mathrm{g} \pm 0.1$ for $18 \mathrm{Ac}$ (Fig. 3). These results indicate that the amount released per unit is equal to the amount of each component in the mixture, and the ratio of the 2 substances was maintained regardless of the difference in vapor pressure of the 2 compounds.

Measuring Pheromone Concentration. Mapping the pheromone plume at 2 distances from the tip of the capillary tube indicated trivial fluctuation in the concentration of $\approx 2 \mathrm{~m}$ at the upwind and downwind end of the tunnel. Furthermore, there was no difference between the pheromone concentration at 50 and 166 $\mathrm{cm}$ from the tip of the capillary tube (Fig. 4). Thus, there is no apparent decline in the pheromone concentration at least $166 \mathrm{~cm}$ downwind from the tip of the sprayer. This confirms our assumption that the aerosol emitted from the tip is completely evaporated as it moves in the downwind direction.

It could be argued that an aerosol might persist in the form of invisibly small droplets moving downwind, especially with solvents or odorants of low vapor pressure. As far as pure solvents that evaporate as bulk fluids at room temperature (e.g., water, hexane, ethanol) are concerned, this can be excluded, because the margin of the aerosol around the tip of the capillary

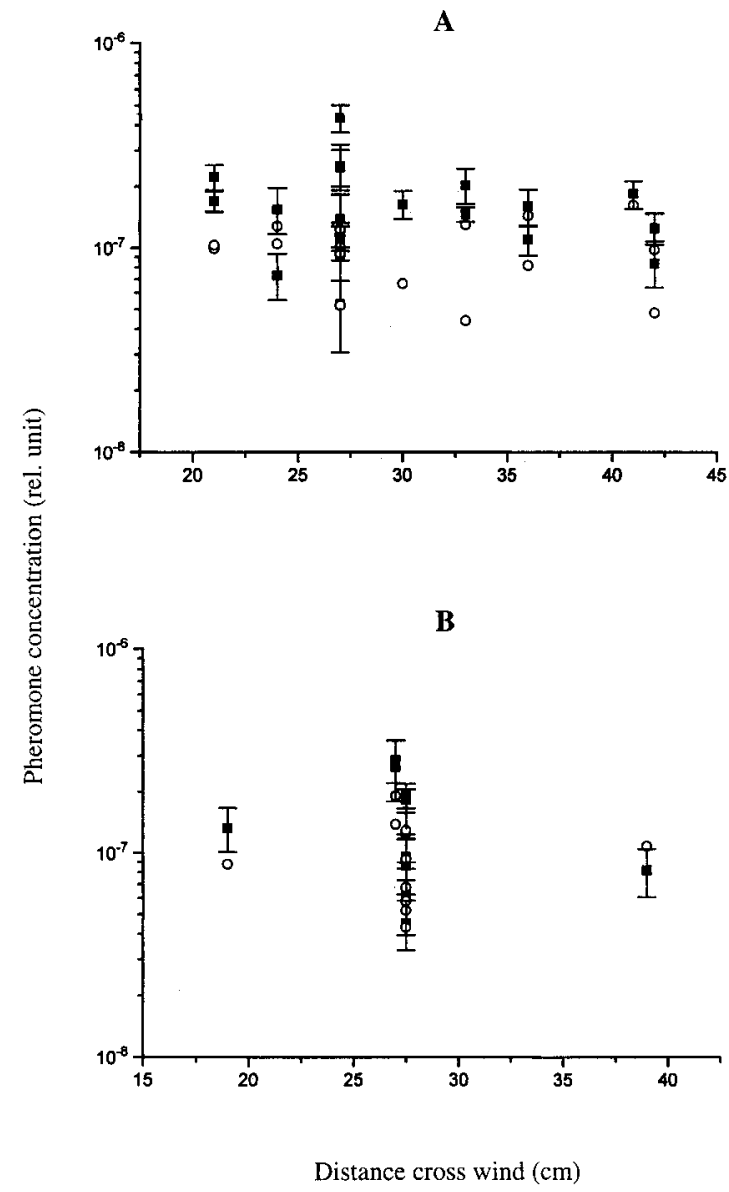

Fig. 4. The EAG measurement of the pheromone concentration at 2 distances from the capillary tip in the wind tunnel. (A) Upwind, $\approx 50 \mathrm{~cm}$ from the tip. (B) Downwind, $\approx 166 \mathrm{~cm}$ from the tip. (口) Measurement from air samples collected from the pheromone plume in the wind tunnel. (○) Measurement from reference samples introduced over the antennae using 3 lateral syringes for calibration.

can be seen to be caused by evaporation of the constituting droplets rather than by their distribution in an increasingly large air-volume. The evaporation of a solvent and a solute are selfaccelerating because the vapor pressure of droplets is proportional to $e^{k / r}(r=$ radius of the droplet, $k=$ constant) (Atkins 1994).

Releasing Different Concentration. Male response was strongly correlated with the release rate of the 3-component blend (Fig. 5). Landing at the source was affected more by release rate than was activation of males. At $0.1 \mathrm{pg} / \mathrm{min}$ of E7,Z9-12Ac, $\approx 38 \%$ of males arrived at the source. When similar amount was loaded on a filter paper or rubber septa, few males were activated and none were able to maintain upwind flight to the source (E.-S., unpublished data). Increasing the release rate to 1,10 and $100 \mathrm{pg} / \mathrm{min}$ led to gradual improvement in all the behavioral sequences of male response (Fig. 5). Maximum landing at the source was obtained at $100 \mathrm{pg} / \mathrm{min}$; further increase of 


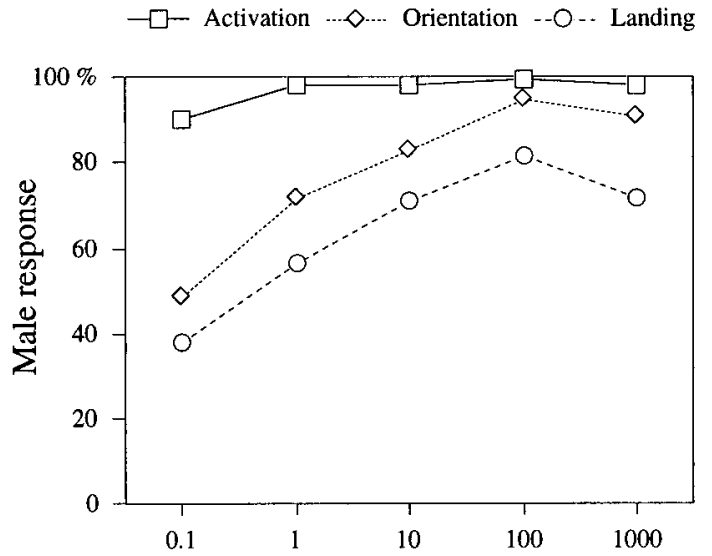

Release rate (pg of E7,Z9-12Ac/min)

Fig. 5. Response of L. botrana males to 4 release rates of the 3-component blend: E7, Z9-12Ac, E7, Z9-12OH, and Z9-12Ac (100: 5: 1).

the release rate to $1,000 \mathrm{pg} / \mathrm{min}$ reduced the number of males arriving at the source but the difference was not significant.

Releasing Pheromone Blends with Proportions of Components. Increasing the proportion of E7,Z9$12 \mathrm{OH}$ to E7,Z9-12Ac in the 2-component blend greatly affected the number of males arriving at the source. Maximum landing was obtained when E7,Z9$12 \mathrm{OH}$ was released at $0.5 \mathrm{pg} / \mathrm{min}$ (Fig. 6). At higher release rates $(2$ and $10 \mathrm{pg} / \mathrm{min}$ ) of $\mathrm{E} 7, \mathrm{Z} 9-12 \mathrm{OH}$, the number of males arrived at the source decreased significantly $\left(\chi^{2}=5.723, \mathrm{df}=1, P<0.0167\right.$ for $2 \mathrm{pg} / \mathrm{min}$, $\chi^{2}=31.64, \mathrm{df}=1, P<0.0001$ for $10 \mathrm{pg} / \mathrm{min}$ ) (Fig. 6). Using filter paper as a release substrate, Witzgall et al. (1993) conducted a similar experiment. However,

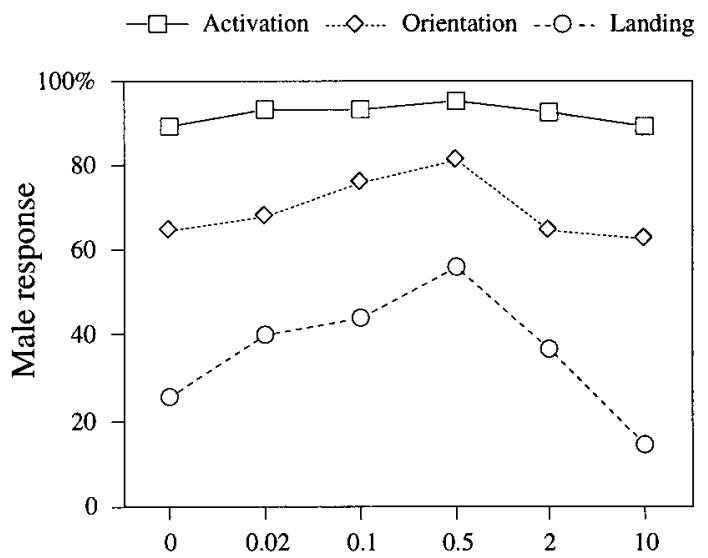

$\mathrm{pg} / \mathrm{min}$ of $\mathrm{E} 7, \mathrm{Z9}-12 \mathrm{OH}$ added

Fig. 6. Response of L. botrana males to 2-component blends with $10 \mathrm{pg} / \mathrm{min}$ of $\mathrm{E} 7, \mathrm{Z} 9-12 \mathrm{Ac}$ plus varying release rate of $\mathrm{E} 7, \mathrm{Z} 9-12 \mathrm{OH}$. they did not observe a behavioral effect of E7,Z9$12 \mathrm{OH}$ at any of the different proportions tested. A tentative explanation for this difference, with the filter paper, as used by Witzgall et al. (1993), the release rate of E7,Z9-12OH did not reflect the initial amount loaded on the filter paper, because E7,Z9-12OH would evaporate quickly, whereas with the sprayer the amount of $\mathrm{E} 7, \mathrm{Z} 9-12 \mathrm{OH}$ released and the ratio to E7,Z9-12Ac over the whole experimental period was constant.

In addition to characterization of the pheromone blend for L. botrana (El-Sayed 1996, 1998a, 1999a), the sprayer has been used successfully in the study of the behavioral affects of (E,E)-8,10-dodecadien-1-ol (E8,E10-12OH) geometrical isomers on sex attraction of C. pomonella (El-Sayed et al. 1998b), the identification of C. pomonella sex pheromone components (El-Sayed et al. 1999b), and is being used for the identification of the sex pheromones of the pea midge, C. pisi (Y. Hillbur and E.-S., unpublished data) and the sex pheromones of E. viteana (R. M. Trimble, personal communication).

The main advantage of the sprayer is that the composition of odor plumes can be set quantitatively and stably over the whole experimental period. This can be achieved using a minimal set of stock solutions, without the restrictions by discrete concentration steps, by purely electronic means (e.g., computer-controlled syringe pump speed) and without the need to control environmental factors. In addition, the use of the sprayer is far more economical than traditional techniques because the odorant is used quantitatively. This is particularly important when using natural extracts or compounds of limited supply.

\section{Acknowledgments}

We thank R. Wehner (Department of Zoology, Zürich University) for his support during this project, U. Koch for providing the EAG system and measuring the pheromone concentration, R. M. Trimble for helpful comment on the manuscript, and 2 anonymous reviewers for critically reviewing the manuscript. This work was partially financed by Bundesministerium für Forschung und Technologie and BASF Company, Project No. 0319459A.

\section{References Cited}

Adam, G., P. Läuger, and G. Stark, [eds.]. 1977. Physikalische chemie und biophysik. Springer, Berlin.

Atkins, P. W. [eds.]. 1994. Physical chemistry, 5th ed. Oxford University Press, Oxford.

Baker, T. C., and R. T. Cardé. 1978. Disruption of gypsy moth male sex pheromone behavior by high frequency sound. Environ. Entomol. 7: 45-52.

Cross, J. H. 1980. A vapor collection and thermal desorption method to measure semiochemical release rates from controlles release formulations. J. Chem. Ecol. 6: 781-787.

El-Sayed, A. 1996. Analysis of behaviour and chemical stimuli in sex attraction of Lobesia botrana (Lepidoptera: Tortricide). Ph.D. dissertation, Zürich University.

El-Sayed, A., P. Witzgall, and H. Arn. 1998a. Location of the pheromone producing gland in the European grapevine 
moth, Lobesia botrana (Lepidoptera: Tortricidae). Appl. Entomol. Zool. 33: 507-511.

El-Sayed, A., R. Unelius, I. Liblikas, J. Löfqvist, M. Bengtsson, and P. Witzgall. 1998b. Effect of codlemone isomers on codling moth (Lepidoptera: Tortricidae) male attraction. Environ. Entomol. 27: 1250-1254.

El-Sayed, A., J. Gödde, P. Witzgall, and H. Arn. 1999a. Characterization of the pheromone blend for the grapevine moth, Lobesia botrana by using flight track recording. J. Chem. Ecol. 25, 2: 389-400.

El-Sayed, A., M. Bengtsson, S. Rauscher, J. Löfqvist, and P. Witzgall. 1999b. Multicomponent sex pheromone in codling moth (Lepidoptera: Tortricidae). Environ. Entomol. 28(5): 775-779.

El-Sayed, A., J. Gödde, H. Arnard. 1999c. Computer-based video system for real-time recording of insect flights in three dimensions (in press).

Färbert, P., U. T. Koch, A. Färbert, R. T. Staten, and R. T. Cardé. 1997. Pheromone concentration measurement with EAG in cotton fields treated for mating disruption of Pectinophora gossypiella (Lepidoptera: Gelechiidae). Environ. Entomol. 26: 1105-1116.

Gödde, J. 1986. A fast voltage-controlled continuous-flow miniature chemostat. J. Neurosci. Methods 16: 191-200.

GraphPad 1993. Instat biostatistics, version 2.0. GraphPad, San Diego, CA.

Kaissling, K. E. 1985. Odorants and their application, pp. 366-368. In A. I. Spielman and J. G. Brand [eds.], Experimental cell biology of taste and olfaction, current techniques and protocols. CRC, Boca Raton, FL.

Linn, C. E., L. W. Bjostad, J. W. Du, and W. L. Roelofs. 1984. Redundancy in a chemical signal: Behavioral responses of male Trichoplusia ni to a six-component sex pheromone blend. J. Chem. Ecol. 10: 1635-1658.
Sanders, C. J. 1990. Responses of male spruce budworm moths to sex pheromone released from filter paper and rubber septa. Can. Entomol. 122: 263-269.

Sauer, A. E., G. Karg, U. T. Koch, J. J. De Kramer, and R. Milli. 1992. A portable EAG system for the measurement of pheromone concentrations in the field. Chem. Senses 17: 543-553.

Shorey, H. H., and R. G. Gerber. 1996. Use of puffers for disruption of sex pheromone communication among navel orangeworm moths (Lepidoptera: Pyralidae) in almonds, pistachios and walnuts. Environ. Entomol. 25: $1154-1157$.

Shorey, H. H., C. B. Sisk, and R. G. Gerber. 1996. Widely seperated pheromone release sites for disruption of sex pheromone communication in two species of Lepidoptera. Environ. Entomol. 25: 446-451.

Abacus Concepts. 1995. StatView for Macintosh, version 4.5. Abacus Concepts, Berkeley, CA.

Treat, A. E. 1962. Comparative moth catches by an ultrasound and a silent light trap. Ann. Entomol. Soc. Am. 55: $716-721$.

Witzgall, P., and H. Arn. 1991. Recording flight tracks of Lobesia botrana in the wind tunnel, pp. 187-193. In I. Hrdy [eds.], Insect chemical ecology. Academia Praha and SPB Academic, The Hague.

Witzgall, P. M. Bengtsson, H. A. Bayer, S. Rauscher, and H. Arn. 1993. Flugwegaufzeichnung als Hilfsmittel für die Identifikation von Schmetterlingspheromonen. Mitt. Dtsch. Ges. Allg. Angew. Entomol. 8: 659-662. 